\section{Re: A Lifestyle Intervention Study in Patients with Diabetes or Impaired Glucose Tolerance: Translation of a Research Intervention into Practice}

To the Editor: I read with interest the research article by Matvienko and Hoehns. ${ }^{1}$ The overarching goal of the study was timely and consistent with the recent paradigm shift toward translational science; furthermore, the authors used a collaborative approach with local health care providers to assist with participant recruitment. I applaud their efforts to connect science with the real world. Although I commend the authors I must comment on their methodology. First, translational science must always be cognizant of the intent of the original science. The specific aim of the Diabetes Prevention Program was to reduce the incidence of diabetes ${ }^{2}$; therefore the current authors' inclusion of known diabetics into a diabetic prevention program negates the intent of the original trial. Also weight gain, which oftentimes is secondary to the diabetic's pharmacotherapy, ${ }^{3}$ potentially is a confounding variable. Alternatively the authors may consider conducting a comparison study which includes 2 groups: one consisting of diabetics and another that does not include diabetics. A design such as this may contribute to the body of literature regarding the effectiveness of the diabetes prevention program in changing anthropometric and physiologic measures in both groups.

Second, the authors stated that they wanted to translate research into a real world practice setting. My conceptualization of a real world setting is one wherein the participant frequents as part of their daily lives. Settings such as recreation departments, community centers, or faith-based environments come to mind. However, the authors conducted the intervention within an academic institution and used trained graduate students to deliver the sessions. Using interventionists who are trained in the intervention is imperative in translational science, because training increases fidelity maintenance. Yet, I don't accept the academic setting as a "real world" translation. Furthermore the academic setting is privy to resources that are not as easily attainable in community settings, such as grant funding. I suggest the use of a more community-based participatory model in which the academic institution partners with the community, trains the community partners, and empowers them to deliver the intervention. Although communities may not be privy to the resources of the academic center, they have their own unique resources. For example, they have community leaders who can be instrumental in providing access to the target population as well as people who are vested in the community and may unselfishly promote health behavior change in their own communities. Oftentimes these individuals are nurses, physicians, educa- tors, and other public/health professionals whose health background enable them to easily grasp the material. Interventions which are conducted using these real world resources are likely to provide the community with sustainable programs that outlast the researchers funding $\operatorname{period}(\mathrm{s})$.

In conclusion, the translation of evidenced-based interventions which target obesity are much needed within community settings. Researchers must be ever mindful of the intent of the original research and equally mindful of the sustainability of resultant successful interventions.

Lovoria B. Williams, MSN, APRN, BC School of Nursing, Medical College of Georgia Augusta, GA lwilliams@mcg.edu

\section{References}

1. Matvienko OA, Hoehns JD. A lifestyle intervention study in patients with diabetes or impaired glucose tolerance: Translation of a research intervention into practice. J Am Board Fam Med 22;5:535-43.

2. Knowler WC, Barrett-Connor E, Fowler SE, et al. Reduction in the incidence of type 2 diabetes with lifestyle intervention or metformin 2002;346:393-403.

3. Krentz AJ, Patel MB, Bailey CJ. New drugs for type 2 diabetes mellitus: What is their place in therapy? Drugs 2008;68:2131-62.

doi: 10.3122/jabfm.2010.02.090239

The above letter was referred to the author of the article in question, who offers the following reply.

\section{Response: Re: A Lifestyle Intervention Study in Patients with Diabetes or Impaired Glucose Tolerance: Translation of a Research Intervention Into Practice}

To the Editor: We appreciate the interest and comments from Ms. Williams ${ }^{1}$ regarding our research article. ${ }^{2}$ She expressed some concern about our adaptation of the Diabetes Prevention Program (DPP) ${ }^{3}$ for a study group that included patients with prediabetes and diabetes. Unlike the Diabetes Prevention Program study, we enrolled patients with diabetes. However, for those patients, enrollment was limited to individuals who were in the early stages of diabetes (diagnosis within the past 5 years) and who had never used insulin. Although including both types of patients somewhat increased the heterogeneity of the study group, we felt that doing so would not unduly compromise the objectives of the study.

It is correctly noted that differences in pharmacotherapy regimens for patients with diabetes can influence patient weight. Five participants started or discontinued 
a diabetes medication during the study. We believe these changes probably had a minor effect on our overall observed anthropometric differences. These and other medication changes that occurred during the evaluation period were summarized.

Although our study was conducted on a university campus, it could have been implemented in any community setting with office space available for meeting with participants. We did not use any special equipment or resources that are unique to the academic setting. Our educational sessions consisted of one-on-one discussion with our participants and periodic assessment of outcomes, including anthropometric measurements and questionnaires. Perhaps the only advantage of having our participants come to campus was that they had access to a fitness area in our building, which we used to conduct cardiovascular fitness assessments and carry out personal physical activity sections to help our participants get started with an exercise program. The majority of our participants chose walking (on a treadmill or track) as their exercise mode and thus did not use any special exercise equipment. We should, however, point out that our campus may be somewhat different from traditional academic settings. Most notably, our university does not contain an academic medical center. It is small $(13,000$ students) and easily accessible to the public. Thus, we consider our campus a "real-world" setting, although we realize that this may not be the case for larger universities.

Translational research studies are a relatively new area of investigation, and the optimal design for these types of studies still requires clarification. Translation of original research into the community setting using exactly the same format may not always be possible or practical. For example, studies that evaluate therapies on disease incidence or progression are usually longitudinal and use a large sample size. This design is not feasible for many practitioners and small communities. Does this mean that findings from large-scale studies should not be translated unless the conditions of the original study are reproduced? We agree with the reader that "researchers must be mindful of the intent of the original research." However, we also believe that there should be some flexibility in how findings are translated depending on individual community needs and resources.

Oksana A. Matvienko, PhD

Department of Physical Education and Leisure Services, School of Health, University of Northern Iowa, Cedar Falls oksana.matvienko@uni.edu

James Hoehns, PharmD, BCPS

Northeast Iowa Family Medicine Residency, University of Iowa College of Pharmacy, Waterloo

\section{References}

1. Williams LB. Re: A lifestyle intervention study in patients with diabetes or impaired glucose tolerance: translation of a research intervention into practice. J Am Board Fam Med 2010;23:273.

2. Matvienko OA, Hoehns JD. A lifestyle intervention study in patients with diabetes or impaired glucose tolerance: translation of a research intervention into practice. J Am Board Fam Med 2009;22:535-43.

3. Knowler WC, Barrett-Connor E, Fowler SE, et al. Reduction in the incidence of type 2 diabetes with lifestyle intervention or metformin. N Engl J Med 2002;346:393-403.

doi: 10.3122/jabfm.2010.02.090279 